\title{
MRI findings in primary brain lymphoma in immunocompetent patients
}

Abstract

Background and objective: Primary brain lymphoma is an extranodal aggressive intracranial neoplasm of lymphocytic origin originating and confined to the brain parenchyma and meninges. It is rare in immune competent patients, but its incidence is increasing. This retrospective study was conducted to record the MRI features of primary brain lymphoma at the time of diagnosis in immunocompetent patients.

Methods: Of the 450 patients diagnosed with the brain tumor during a period of five years from 2008 to 2013, the clinical features and MRI findings of 16 cases of pathologically proven to be non-Hodgkin's lymphoma were studied. All the patients were tested negative for HIV and there was no history of immune suppression drugs or any other chronic illness. All the patients were examined with MRI observing the tumor location, multifocality, signal intensity in different sequences, enhancement patterns, peritumoral edema, the presence of hemorrhage and calcification.

Results: Of the 16 patients, including the monofocal and multifocal cases, 30 lesions exhibited. The mean age at diagnosis was 53 years. Nine patients $(56.25 \%)$ found to have a multifocal disease. In more than $75 \%$ of lesions, MRI was hypo to iso signal on T1 and T2. Mild to moderate perilesional edema, strong contrast enhancement and restricted diffusion were seen in all cases. The hemorrhagic tumor was noticed in four lesions (13.3\%). No calcification and no leptomeningeal lesions were noted. The MRI images in post steroid therapy were studied within one month of treatment. Tumour regression was noticed in 21/30 (70\%), stable in 3/30 (10\%) and progressing in 6/30 (20\%).

Conclusion: MRI is a reliable imaging technique in the management of patients with primary brain lymphoma. Early accurate diagnosis is crucial to avoid the unnecessary operation and shift patients from extensive surgery to chemoradiotherapy.
\end{abstract}

Keywords: MRI; Brain Lymphoma; immune competent; Tumor regression

\section{Introduction}

Primary brain lymphoma is a rare variant of non-Hodgkin's lymphoma; it accounts for $6 \%$ of all primary brain tumours ${ }^{1}$. Microscopically $90 \%$ of primary central nervous system lymphomas are of B cell type while, the poorly characterized low grade, Burkitt's or T cell lymphomas formed the remaining $10 \% .^{1,2}$ Among immune competent patients primary brain lymphoma is more common in male, typically presents about the age of fifty and most common presenting symptoms at the time of diagnosis are focal neurological deficits, neuropsychiatric symptoms, symptoms related to increasing intracranial pressure, epilepsy and visual disturbances. ${ }^{3}$ Despite the characteristic imaging features of brain lymphoma on CT and MRI but it is not always possible to differentiate it from other neoplastic or nonneoplastic brain conditions. This may be due to overlap in the imaging findings, or due to lack of the typical imaging characteristics. DWI, perfusion MR imaging, and MR spectroscopy are more frequently used in practice, and they add more specific signs that could differentiate lymphomas from other lesions of the brain. ${ }^{4}$ Currently, there are no established

* Department of Surgery, College of Medicine, Hawler Medical University, Erbil, I raq. 
imaging biomarkers that are prognostic of clinical outcome in patients with primary brain lymphoma. The pre therapeutic tumor apparent diffusion coefficient (ADC) measurements may noninvasively predict clinical outcome in patients with primary lymphoma of CNS. ${ }^{5}$ Low ADC values were predictive of shorter progression-free survival and overall survival. In addition, an inverse correlation was found between ADC values and the cellular density of the tumors. Patients with prolonged progression-free survival and overall survival also had a significant reduction in post-therapeutic ADC values. Thus, repeated ADC measurements may be used as biomarkers in the surveillance of therapeutic response. ${ }^{4}$ Diagnostic workup should start with contrast enhanced brain MRI, which is the most sensitive imaging method to detect primary central nervous system lymphoma. Further diagnostic steps include cerebrospinal fluid analysis and slit lamp eye examination especially when the patient complains of ocular symptoms. For definite diagnosis, the patient should finally undergo stereotactic biopsy. ${ }^{6}$ This study aimed to depict the MRI findings of primary brain lymphoma during initial diagnosis in immunocompetent patients to facilitate differentiation of central nervous system lymphoma from other brain neoplasms.

\section{Methods}

This study was carried out in Erbil, Kurdistan Region of Iraq over five year period from October 2008 to November 2013. Four hundred and fifty cases of brain tumors were retrospectively studied of which sixteen patients proved pathologically as having primary brain lymphoma. All patients tested negative for HIV or any other immunosuppressive disorder. All of them proved to be disease free outside the brain. All cases were analyzed by age, sex, clinical presentation and MRI features.

Image protocol: MR imaging unit $1.5 \mathrm{~T}$ Siemens Symphony, Erlangen, Germany was used. The standard MRI images included Axial spin echo (SE) T1- weighted images (T1WI), axial fast spin echo (FSE) T2- weighted images (T2WI), axial fluid attenuated recovery FLAIR, Axial, coronal, and sagittal post contrast (gadolinium) spin echo (SE) T1WI and diffusion weighted images (DWI) with $b$ value of 0,500 and $1000 \mathrm{~s} / \mathrm{mm}^{2}$. Slice thickness was $5 \mathrm{~mm}$ and Field of view (FOV) was $230 \mathrm{~mm}$. Signal intensity of the tumor in T1WI, $\mathrm{T} 2 \mathrm{WI}$, and post contrast images were studied as well as the tumor margins if it is infiltrative, irregular or well demarcated. The location, multiplicity of lesions and perilesional edema were also studied. Nine patients had follow-up MRI examinations after steroid therapy within one month from the commencement of the treatment and tumor volume measurement done to assess the follow-up. Tumor volume was measured on contrast enhanced T1WI by summation of each area $\left(\mathrm{mm}^{2}\right)$ multiplied by slice thickness. The tumor was labeled regressed if there were more than 13\% reduction in its volume, stable if the change in the volume was less than $13 \%$ and progressing if the tumor volume increased by more than $13 \%$, marginal recurrence or development of new lesion. ${ }^{7}$ All patients underwent histopathological and immunohistochemisty confirmation as low to high grade non Hodgkin's lymphoma. All the data were statistically analyzed using Excel program. This study was approved by the Research Ethics Committee of Hawler Medical University in Erbil, Iraq and informed consent was obtained from participants.

\section{Results}

The age range was from 28 to 65 years with a mean age at diagnosis was 53 years. The commonest age group was 50 60 years. The youngest patient was 28 years old. There were 12 males and four females (male: female 3:1). The median time between the onset of symptoms and diagnosis was two months. Its incidence was $3.5 \%$ among all intracranial tumors. 
https:/ / doi.org/ 10.15218/ zjms.2017.032

The presenting symptoms in decreasing order were headache $(14 / 16 ; 87.5 \%)$, neurological deficit (12/16; 75\%), vomiting (8/16; 50\%), confusion (5/16; 31.25\%), seizures $(3 / 16 ; 18.75 \%)$ and gait ataxia (1/16; 6.25\%). The most common locations in decreasing order were cerebral hemispheres $(43.75 \%)$, in particular, the frontal and parietal lobes, deep structures like basal ganglia and corpus callosum (30\%), trancallosal infiltration (crossing midline) were seen in (18.75\%)and cerebellum (6.25\%). Nine of our patients (56.25\%) were found to have multiple lesions in their pretreatment images. There were total 30 lesions in 16 patients. The signal changes are low signal intensity in T1 in (12/30; $40 \%$ ), iso intense to gray matter in T1WI in $(14 / 30 ; 46.6 \%)$ and slightly high signal intensity in T1WI in $(4 / 30 ; 13.3 \%)$. The signal intensity in T2WI was low in (10/30; $33.3 \%)$, iso intense to gray matter in $(13 / 30 ; 43.3 \%$ and slightly high signal intensity in T2 (7/30; 23.3\%). The entire lesion (30/30; 100\%) were highlighted in FLAIR and showed restricted diffusion with low signal intensity in ADC (Table 1). All the lesions were associated with perilesional edema as noticed on FLAIR sequence. All the lesions showed mild to moderate enhancement. Four of the lesions showed evidence of hemorrhage. None of the lesions on imaging had evidence of calcification. Intraventricular, and subependymal and leptomeningeal infiltration was not seen in any case. The margins of the lesions were well defined in $(4 / 30 ; 13.3 \%)$, irregular in $(16 / 30 ; 53.3 \%)$ and infiltrative in $(10 / 30 ; 33.3 \%)$. (Figures $1,2,3,4$ and 5 show the various MR signal changes). The MRI images in post steroid therapy were studied within one month of treatment. Tumour regression were noticed in $(21 / 30 ; 70 \%)$, stable in $(3 / 30 ; 10 \%)$ and progressing in $(6 / 30 ; 20 \%)$.

Table 1: MRI findings in primary brain lymphoma.

\begin{tabular}{lccccc}
\hline MRI sequences & Low & Iso to gray matter & High & Restricted & total \\
\hline T1 & 12 & 14 & 4 & ------ & 30 \\
T2 & 10 & 13 & 7 & ------- & 30 \\
FLAIR & 0 & 0 & 30 & -------- & 30 \\
DWI & ------ & ------ & ---- & 30 & 30 \\
Contrast enhancement & ------- & ------- & ------ & 30 & 30 \\
\hline
\end{tabular}

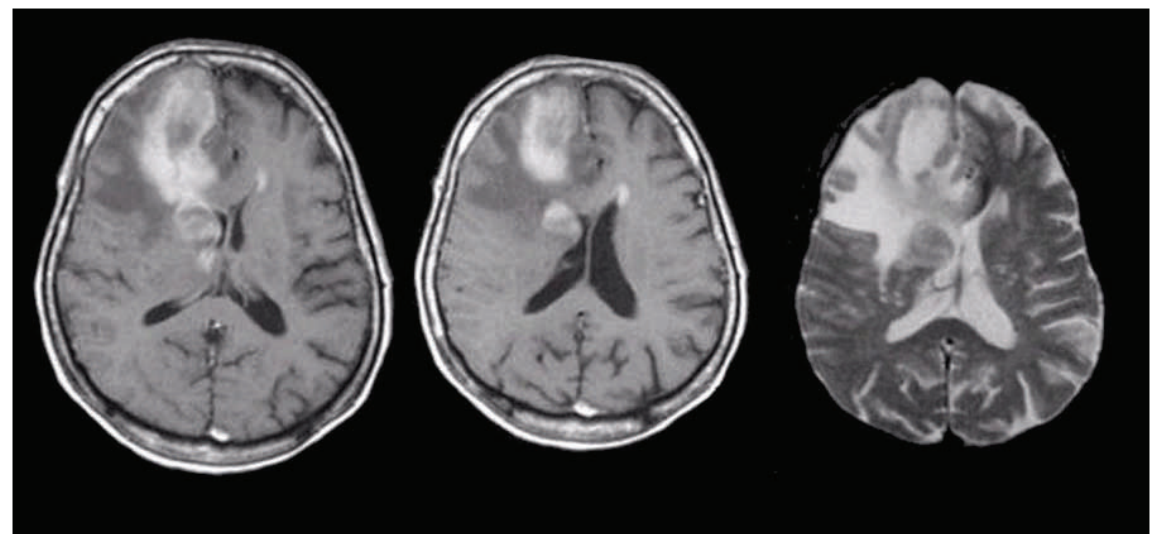

Figure 1: Left and Middle T1-weighted after contrast axial MRIs; (Right) T2-weighted axial MRI. Large enhancing mass involving the right frontal lobe and the basal ganglia with surrounding edema is seen. Biopsy demonstrated lymphoma. 

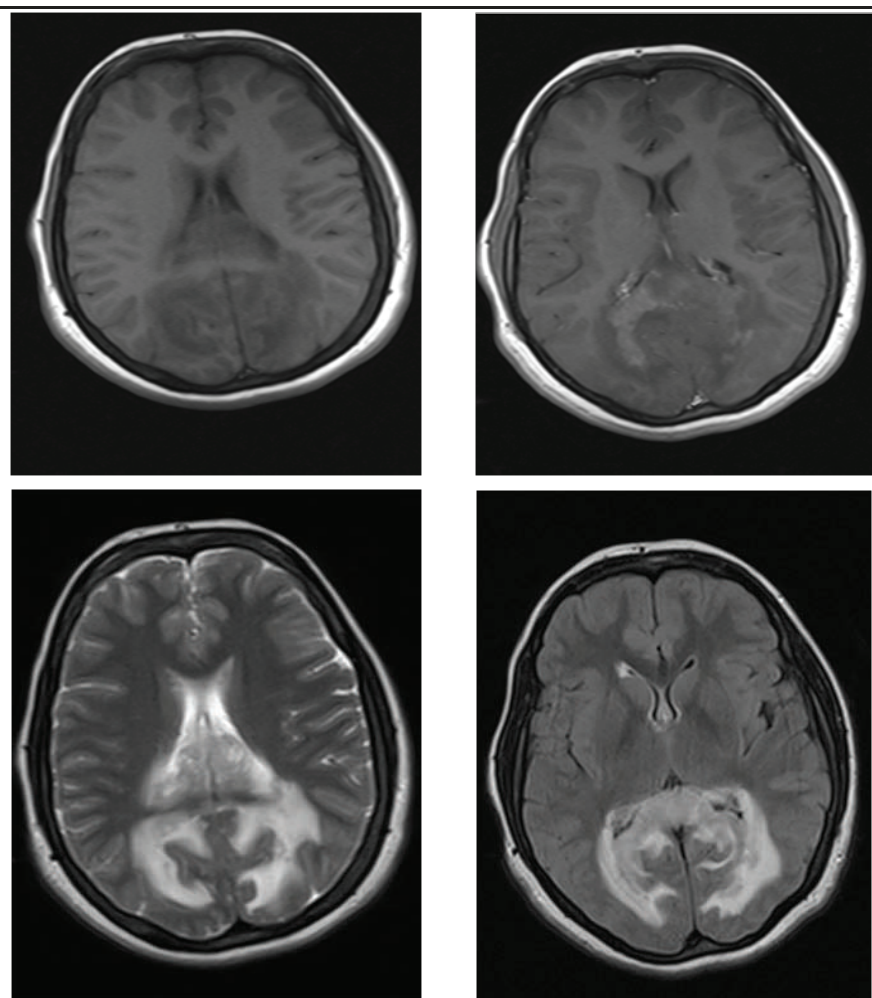

Figure 2: Upper row: Left and right T1W and T1W post contrast MRIs; Lower row left T2W axial, Right is FLAIR axial MRI showing large enhancing mass in the corpus callosum crossing the midline (butterfly appearance) surrounded by edema. Biopsy demonstrated lymphoma.
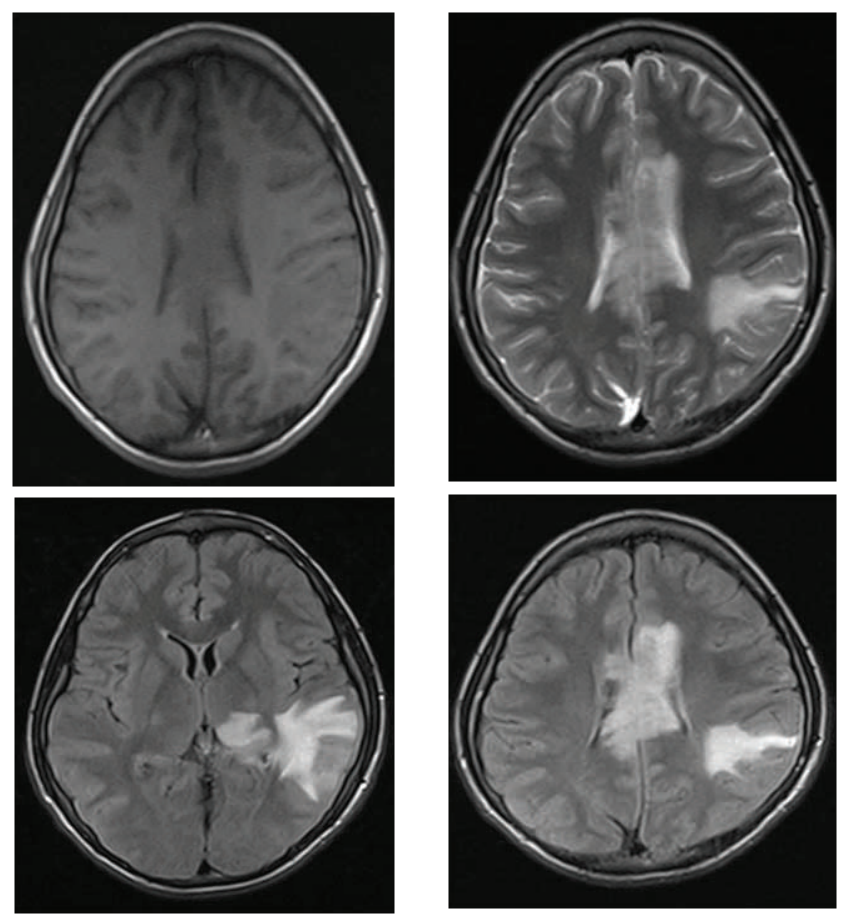

Figure 3: T1W, T2W and FLAIR images showing multifocal lesions in the left temporal, parietal lobes and the corpus callosum also lesion is seen deeply seated in left thalamus. 
Figure 4: DWI showing restricted diffusion in lymphoma.
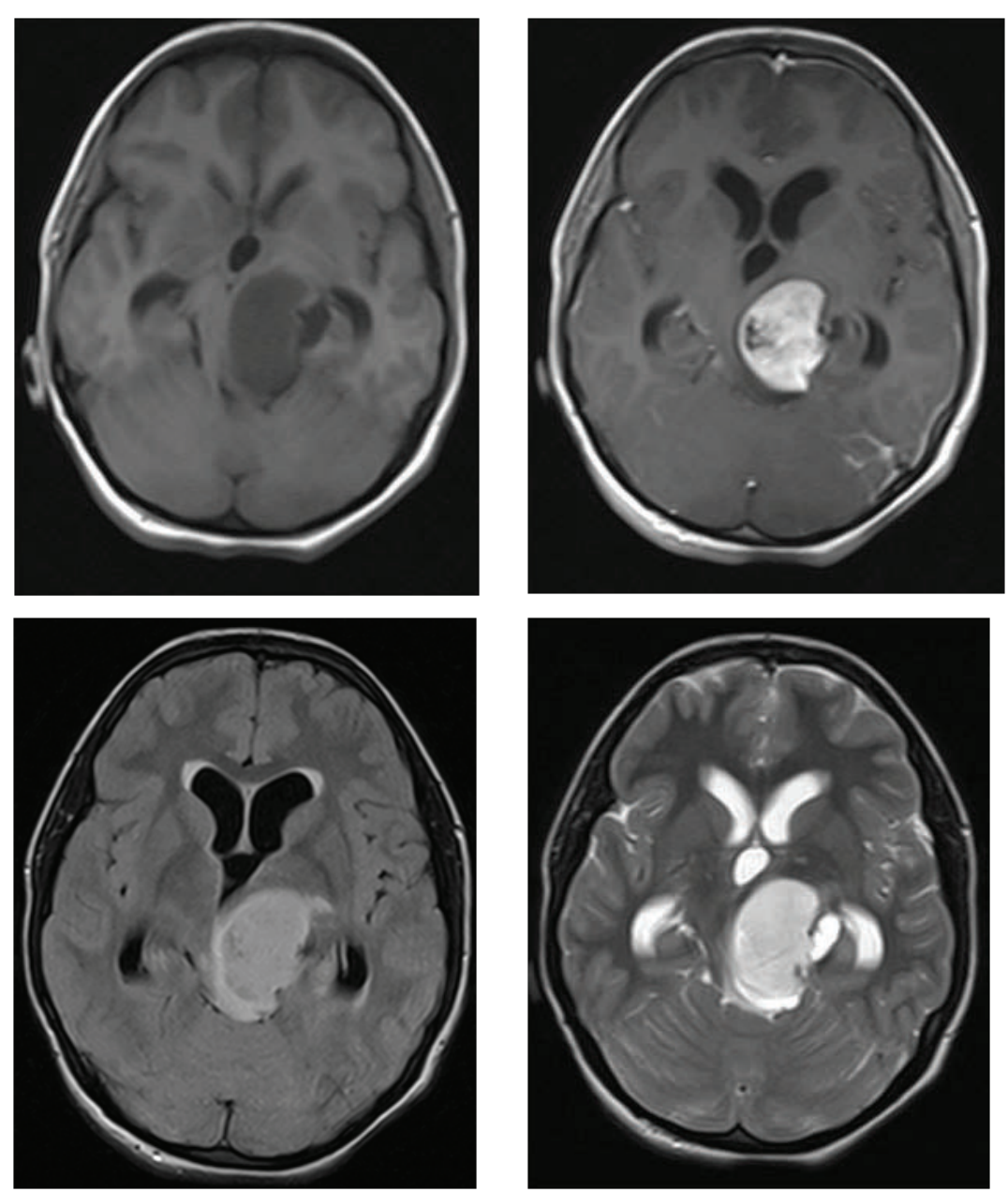

Figure 5: Upper row: Left and right T1W and T1W post contrast MRIs; Lower row left FLAIR axial, right T2W axial MRI showing large enhancing mass in left thalamus with mass effect. The lesion is low in T1W, intermediate in T2W. Biopsy demonstrated lymphoma. 


\section{Discussion}

Primary central nervous system lymphoma is a rare brain tumor, primarily seen in immunocompromised patients (e.g., AIDS, organ transplantation). Its incidence is increasing $(3 \%$ of all primary brain tumors). Patients present with headaches, focal neurological deficits or seizures. Supratentorial lesions are commoner than infratentorial ones. The tumor can involve the deep white matter, the meninges, and the eye. If it involves the deep white matter, it may spread across the corpus callosum, similar to the pattern seen in glioblastoma multiforme. ${ }^{8}$ Tumours appear solid, rarely there are liquefied components, and are iso intense to normal brain tissue in T2WI and T1WI in about two third of cases and usually homogeneously enhancing. Mild to moderate peritumoural edema is common in primary brain lymphoma and is generally less severe than in metastases and high grade glioma. The fact that peritumoural edema is less common is due to infiltrative nature of the tumour. ${ }^{9}$ Multiple lesions are commonly seen in about $50 \%$ of cases; ${ }^{10}$ these are almost in line with the results of a current study. Buhring et al. found that cerebral parenchymal infiltration with lymphoma was about $31 \%$, corpus callosum $15 \%$, basal ganglia $15 \%$ cerebellum $14 \%$ and leptomeningeal infiltration scored $7 \%$, the findings that are close to the results of this work except that we could not detect any leptomeningeal involvement in our work. ${ }^{11}$ In metastatic lymphoma of the central nervous system, it is exceptional for parenchymal masses to be seen without leptomeningeal involvement. This point may help in differentiating between primary and secondary central nervous system lymphoma. ${ }^{8}$ Jack et al. stated that lymphomatous masses do not calcify and hemorrhage is distinctly uncommon on imaging studies and this is similar to our results. ${ }^{12}$ As for post gadolinium enhancement, our data and previous reports support that the lesions typically enhance homogeneously. Less commonly a peripheral pattern of enhancement is noted in immune competent patients. ${ }^{13}$ Rapid regression after steroid therapy was noted in about $70 \%$ of cases and this is in accord with studies done by others. Metastases and glioblastoma multiformis cannot have such rapid response. The explanation includes tumorlysis secondary to steroid therapy or cyclic biological activity. ${ }^{14}$ Unusual manifestations of primary brain lymphoma may make the diagnosis with conventional MRI and CT difficult and thus special sequences and post processing techniques may be obtained for further information to at least narrow the differential diagnosis. Furthermore, cross-sectional imaging might be of benefit for treatment response assessment. ${ }^{15}$ On perfusion imaging, there is usually lower perfusion in primary CNS lymphoma than other brain tumors such as high grade glioma and metastases. The low perfusion in combination with the strong enhancement in primary CNS lymphoma is explained by low induction of neovascularization and destruction of the blood brain barrier. Diffusion imaging with ADC map is commonly performed protocols owing to their short scan time and usefulness in problem solving, and restricted diffusion with low ADC map is typical for primary CNS Iymphoma. ${ }^{15,16}$

\section{Conclusion}

MRI is the imaging modality of choice in the evaluation of suspected primary central nervous system lymphoma. Primary brain lymphoma could be single or multiple lesions. The imaging features depend on the immune status of the patient. In immune competent patients, it typically presents as an enhancing mass in the periventricular region often crossing the midline through the corpus callosum to involve both hemispheres. Involvement of the basal ganglia and the thalami is also common.

\section{Conflicts of interest}

The authors report no conflicts of interest. 


\section{References}

1. De Belder F, Van Cauter S, van den Hauwe L, Van Hecke W, Emsell L, De Belder M, et al. DTI in diagnosis and follow-up of brain tumours. In: Diffusion Tensor Imaging. New York: Springer; 2016. Available at http.springer.com/ chapter/10.1007\%2F978-1-4939-3118-715,2016

2. Shenkier TN, Blay JY, O'Neill BP, Poortmans P, Thiel E, Jahnke $\mathrm{K}$, et al. Primary CNS lymphoma of T-cell origin: A descriptive analysis from the international primary CNS lymphoma collaborative group. J Clin Oncol 2005; 23: 2233 -9 .

3. Olson JE, Janney CA, Rao RD, Cerhan JR, Kurtin PJ, Schiff D, et al. The continuing increase in the incidence of primary central nervous system non-Hodgkin lymphoma: a surveillance, epidemiology, and end results analysis. Cancer 2002; 95: 1504-10.

4. Haldorsen IS, Espeland A, Larsson EM, Central nervous system lymphoma: characteristic findings on traditional and advanced imaging, AJNR 2011:32:984-92.

5. Barajas RF, Rubenstein JL, Chang JS. Diffusion weighted MR imaging derived apparent diffusion coefficient is predictive of clinical outcome in primary central nervous system lymphoma. AJNR 2010; 31: 60-6.

6. Coulon A, Lafitte F, Hoang-Xuan K, Martin-Duverneuil N, Mokhtari K, Blustajn J, et al. Radiographic findings in 37 cases of primary CNS lymphoma in immunocompetent patients. Eur 2002; 12:329-40.

7. Therasse P, Arbuck SG, Eisenhauer EA, Wanders $\mathrm{J}$, Kaplan RS, Rubinstein L, et al. New guidelines to evaluate the response to treatment in solid tumors. J Nat Cancer Inst 2002; 92(3):20516.

8. Zhang D, Hu LB, Henning TD, Ravarani EM, Zou LG, Feng XY, et al. MRI findings of primary CNS lymphoma in 26 Immunocompetent patients. KJR 2010; 11(3):269-77.

9. Alaa EL, Iman Z. primary central nervous system lymphoma: incorporating MRI in the planning of treatment strategies. J Egyptian Nat Cancer Inst 2001; 13 (3): 237-44.

10. Russel DS, Rubinstein LJ, pathology of tumors of nervous system. $5^{\text {th }}$ ed. Baltimore: Williams and Wilkins; 1989.

11. Buhring $U$, Herrlinger $U$. MRI features of primary central nervous system lymphoma at presentation. Neurology 2001; 57 (3): 393-6.

12. Jack CR, O'Neil BP, Banks PM, Rse DF: central nervous system lymphoma: histological types and CT appearance. Radiology1988; 167: 211-5.

13. Osborn AG. Diagnostic neuroradiology. St. Louis, MO: Mosby; 1994.
14. Rumana M, Khursheed N, Ahmad R, Altaf K, Altaf R ,Muhammad B K, et al. Primary CNS Lymphoma in Immunocompetent: A Review of Literature and Our Experience from Kashmir. Turkish Neurosurgery 2011; 21(1): 39-47.

15. Partovi S, Karimi S, Lyo JK, EsmaeiliA, TAN J, Deangelis LM. Multimodality imaging of primary CNS lymphoma in immunocompetent patients. Br J Radiol 2014; 87(1036): 20130684.

16. Horger $M$, Fenchel $M$, Nagele $T$, Moehle $R$, Claussen CD, Beschorner R, et al. Water diffusivity: comparison of primary CNS lymphoma and astrocytic tumor infiltrating the corpus callosum. AJR 2009; 193: 1384-7. 